\title{
Migrationsgesellschaftliche Erinnerungsorte auf heimatkritischer Grundlage. Konturen eines Didaktisierungskonzeptes für die Erwachsenenbildung DaF/DaZ
}

Spaces of memory in migration society in the context of the concept of home criticism. Shapes of a didactical concept for German as a foreign and second language in adult education

Johannes Köck

\begin{abstract}
This paper aims to outline a dissertation project, which was presented at the conference Aktuelle landeskundliche Ansätze in DaF/DaZ (Actual cultural studies on didactic in German as a foreign language and second language). Furthermore, I want to conceptualize two terms, that focus to this perspective - migrationssensibel (migrational sensitive) and heimatkritisch (home critic). The paper starts with a short historical overview and a debate on the role of the concepts of Landeskunde and Erinnerungsorte (Spaces of memory). It also presents the methodological design that will be used for this dissertation project (Grounded Theory, and City Walks). Finally, I will describe the expected outcome of this dissertation project.
\end{abstract}

\section{Keywords}

Cultural Studies; Migration Pedagogy; Migration Society; Spaces of Memory; German as a Foreign and Second language; Grounded Theory; City Walk; Home Critisicm; Migration Sensitivity 


\section{Problemaufriss}

Das Dissertationsprojekt Migrationsgesellschaftliche Erinnerungsorte auf heimatkritischer Grundlage. Konturen eines Didaktisierungskonzeptes für die Erwachsenenbildung DaF/DaZ, will bislang wenig beachtete migrationsgesellschaftliche Erinnerungsorte didaktisch fruchtbar machen und darüber hinaus Konturen einer heimatkritischen und migrationssensiblen Landeskunde generieren. Dieser Beitrag ist angelehnt an ein Exzerpt, welches im Kontext der Anmeldung meines Dissertationsthemas an der Universität Wien entstanden ist. ${ }^{1}$ Um die Relevanz und allgemeine Problemstellung meines Vorhabens illustrieren zu können, ist es zunächst notwendig, die historischen und gegenwärtigen Entwicklungslinien der beiden zentralen Konzepte Erinnerungsort und Landeskunde zu skizzieren und anschließend in ein Verhältnis zum Forschungsdesiderat der Arbeit zu setzen.

\section{Landeskunde}

Hans Simon-Pelanda (2001: 48) verwendet zur Darstellung landeskundlicher Entwicklungslinien die Metapher von „Pendelschwungbewegungen“, innerhalb derer exklusiv behauptete Ansätze zwar aufeinander abfolgen, aber gleich dem Pendel einer Uhr stets auch vice versa, also in die andere (Schwung-)Richtung schlagen. Folgt man diesem Bild, so scheint eine synchrone, lineare Darstellungsweise mit klar voneinander abgrenzbaren Phasen und Epochen zumindest problematisch, wenn nicht gar unmöglich. Dennoch findet sich in älteren Darstellungen (vgl. etwa Christ 1979, oder Neuner 1994) die Schilderung einer fremdsprachenwissenschaftlichen Teildisziplin, die trotz inhaltlicher Kontroversen eine lineare Progression von klassischen zu aktuellen Ansätzen aufweist (vgl. Koreik - Pietztuch 2010: 1444). Zwangsläufig ist jeder Versuch einer historischen Systematisierung und chronologischen Darstellung landeskundlicher Ansätze und Konzepte aber ein konstruierender Vorgang, der beeinflusst ist von inhaltsrelevanten Entscheidungen und Auslassungen sowie Interpretationen (vgl. ebd.: 1441), die transparent gemacht werden müssen. Sich dieses Umstands und der Simplifizierung historisierender Erzählstränge bewusst, soll hier dennoch ein kurzer historischer Überblick vorgenommen werden, der nicht nur die Relevanz meiner Themenstellung sichtbar und nachvollziehbar macht, sondern diese gleichzeitig auch diskursiv verortet. Denn trotz einer häufig ausufernden landeskundlichen Debatte besteht in der Fremdsprachendidaktik seit langem ein breiter Konsens darüber, dass fremdsprachige Fertigkeiten und Kenntnisse verknüpft sein müssen mit einer inhaltlichen Beschäftigung der gesellschaftlichen Wirklichkeiten eines Zielsprachenlandes sowie mit einer Thematisierung historischer und kultureller Fragen (vgl. ebd.). Trotz dieser Einigkeit hält Adelheit Schumann in ihrer Definition gerade die kontroverse und sich häufig ändernde Gegenstandsbestimmung

1 Die Arbeit wird von Univ.-Prof. Dr. İnci Dirim, M.A. von der Universität Wien und Prof. Dr. Claus Altmayer vom Herder-Institut der Universität Leipzig betreut. 
als wesentlich für das Fach Landeskunde fest (Schumann, 2010). Sichtbar wird dieser Aushandlungsprozess in dem seit jeher und nach wie vor bestehenden terminologischen Streit, aus dem eine Vielzahl alternativer Bezeichnungen hervorging. Die Schwierigkeit einer begrifflichen Bestimmung verdeutlicht den omnipräsenten Wunsch nach typologischer Klarheit und findet sowohl im internationalen als auch im deutschsprachigen Diskurs seinen Niederschlag. Aus dem terminologischen Aushandlungsprozess resultieren etwa die folgenden Bezeichnungen:

- Kulturstudien/Kulturkunde

- Leutekunde

- Landesstudien/Landeswissenschaft

- Kulturwissenschaft/ Kultur- und Landeswissenschaft (vgl. Koreik - Pietztuch 2010: 1442).

Trotz dieser Vielzahl an Termini ist seit den 1960er Jahren Landeskunde der dominierende Begriff, der in neueren Fachdebatten allerdings häufig im Zusammenhang mit Bezeichnungen wie: interkulturell, pragmatisch, sozialwissenschaftlich, implizit, explizit verknüpft wird (vgl. ebd.). Diesem gängigen Vorgehen folgt auch mein Dissertationsprojekt, welches die Notwendigkeit einer migrationssensiblen und heimatkritischen Landeskunde darlegen will und trotz berechtigter Kritik (vgl. ebd.) am Terminus Landeskunde an diesem etablierten Begriff festhalten will, allerdings unter einer grundlegend anderen Konzeptualisierung. Das geschilderte Bezeichnungsproblem ist nicht lediglich ein Kampf um (den) einen richtigen Begriff, es ist vielmehr auch Ausdruck einer inhaltsbezogenen Debatte um angemessene Ansätze, Methoden und Konzepte. Aus diesem Grund können die internationalen Termini auch nicht einfach ins Deutsche übersetzt werden, weil sie sich auf unterschiedliche theoretische, bildungs- und gesellschaftspolitische Begründungszusammenhänge beziehen (vgl. Koreik - Pietztuch 2010: 1442). Nachdem die Schwierigkeit einer begrifflichen Bestimmung skizziert wurde, sollen jetzt die groben Entwicklungsstränge landeskundlicher Themen in einem historischen Überblick wiedergegeben werden. Das Prinzip der Realienkunde war die letzten drei Jahrzehnte des 19. Jahrhunderts ein Mittel zur Betonung utilitaristischen Wissens über „Land und Leute" und sah sich als Gegensatz zu einem vorherrschenden altphilologischen Prinzip, bei dem einzig Übersetzung alter Klassiker und Grammatikvermittlung als höchstes Ziel galt (vgl. ebd.: 1445). Im Sinne einer positivistischen Denktradition sollten nunmehr wichtige geographische ökonomische und geschichtliche enzyklopädische Fakten vermittelt werden, um die Lernenden über Realien anderer Länder aufzuklären. Der Begriff Realienkunde $e^{2}$ kann somit ein realhistorisches Prinzip darstellen, wird aber auch in aktuellen fremdsprachlichen Diskursen, etwa in Tschechien verwendet, wenn von der Vermittlung faktischen Wissens die Rede ist. So gibt es etwa an der Masaryk-Universität das Kursangebot Německé reálie a jazyková cvičenî3 Die Kulturkunde löste mit Beginn des 20. Jahrhunderts die Realienkunde ab und stellte ein kulturvergleichendes Paradigma in den

2 Zentrale Begriffe sind kursiv gesetzt.

3 Übersetzt mit German Cultural Studies and Language Practice (Vgl. https://is.muni.cz/course/ped/ spring2020/NJ081, letzter Zugriff: 8. 1. 2020). 
Mittelpunkt, wobei nicht mehr nur enzyklopädisches Wissen vermittelt werden sollte, sondern die „fremde Kultur“4 sollte im Kontrast zur „eigenen Kultur“ betrachtet und in ihrer Ganzheit verstanden werden. Dieses Ergründen-Wollen des „fremden Volkscharakters“ war von Anfang an dominiert von stereotypisierenden und rassialisierenden Zuschreibungen, die das „deutsche Wesen“ kontrastiv zu einem sogenannten Wesen „anderer Völker“ versteht und ab den 1930er Jahren gar in eine „nationalistische Wesenskunde“ mündete (vgl. Koreik - Pietztuch 2010: 1442; Weimann - Hosch 1993). Sowohl die Kultur- als auch die Realienkunde können somit als Beispiel für ideologischen Missbrauch dienen. Der Begriff Landeskunde hat sich seit den späten 1960er Jahren trotz vielfältiger Kritik bis heute hartnäckig gehalten. Dies liegt einerseits an der gewünschten Abgrenzung zum vorbelasteten Begriff „Kulturkunde“ und andererseits an der „Vielfalt und Variationsbreite dessen, was unter dem Begriff schrittweise subsumiert und entwickelt wurde“ (Koreik - Pietztuch 2010: 1446). Weimann und Hosch führten 1991 die begriffliche Unterscheidung zwischen einem kognitiven (faktischen), kommunikativen und interkulturellem Ansatz ein, die neben dem seit den 2000er Jahren bestehenden kulturwissenschaftlichen Zugang bis heute Gültigkeit besitzt. Zwar ist diese Unterscheidungspraxis natürlich schemenhaft und in praktischen Kontexten treten häufig hybride Formen auf, dennoch ist sie als Modell hilfreich. Demnach hat der Kognitive Ansatz ähnlich der Realienkunde einen enzyklopädischen Anspruch und ist folglich abhängig von den Bezugswissenschaften. Landeskunde soll hier ein eigenes, nicht in den Sprachunterricht integriertes Fach sein. Kritisiert wird der kognitive Ansatz zu Recht für seinen mangelnden Praxisbezug, eine ebenso mangelnde Lernenden-Zentriertheit und die Vermittlung von stereotypisierendem, deklarativem Wissen. Auch die fehlende Integration in den Sprachunterricht wird kritisiert. Eine reziproke Ausrichtung verfolgt der Kommunikative Ansatz, der die Landeskunde komplett in den Dienst des sprachlichen Handelns stellt und auch die Themen nach der Alltagsrelevanz wählt. Die Fremdsprache ist Instruktionssprache und wird von Anfang an verwendet. Trotz der offensichtlichen Fortschritte gegenüber dem kognitiven Ansatz bleibt das Problem eines Universalitätsanspruchs sowie die Ausklammerung der Erstsprachen bzw. jeglicher anderer sprachlicher Ressourcen und eine starke germanozentrische Perspektive als Kritikpunkte (vgl. Weimann Hosch 1993). Spätestens seit den späten 1980er Jahren und den ABCD-Thesen stehen interkulturelle landeskundliche Zugänge im Fokus. In besagten Thesen wurde Landeskunde als ein Prinzip gefasst, welches sich durch die Kombination von Sprachvermittlung und kultureller Information konkretisiert. Landeskunde bezieht sich demnach auf den gesamten deutschsprachigen Raum mit nicht nur nationalen, sondern auch regionalen und grenzübergreifenden Phänomenen. „Primäre Aufgabe der Landeskunde ist nicht die Information, sondern Sensibilisierung sowie die Entwicklung von Fertigkeiten im Umgang mit fremden Kulturen“ (ABCD-Thesen 1990: 26). Interkulturelle Landeskunde strebt nach Toleranzentwicklung, dem Auseinandersetzen und Verstehen der „fremden Kultur“ und will zum Kulturvergleich sowie zum interkulturell kompetenten Handeln anregen. Altmayer (2006: 46) betont zu Recht, die positiven Aspekte der Ein-

4 Die Setzung von „" dient hier der Markierung der simplifizierenden und zuschreibenden Implikationen der Termini, etwa: „eigen“ vs. „fremd“. 
beziehung der eigenkulturellen, handlungsbezogenen Perspektive der Lernenden im Vergleich zu einer vormals fakten- und wissensorientierten Landeskunde, nennt aber auch erhebliche Schwächen und Probleme des interkulturellen Ansatzes. Zunächst ist der Begriff interkulturell nicht trennscharf, sondern wird in einer Vielzahl von Kontexten und teils synonym verwendet, obwohl sich hinter der identen Bezeichnung häufig sehr verschiedene Konzepte von Interkulturalität verbergen (vgl. ebd.). Hauptkritikpunkt Claus Altmayers ist aber das häufig implizit zugrunde gelegte essentialistische und homogenisierende Kulturverständnis nach dem es sich bei Kulturen „um reale, nach außen mehr oder weniger klar abgegrenzte und nach innen mehr oder weniger homogene Gruppen von Menschen handelt, die objektiv bestimmte Gemeinsamkeiten des Verhaltens, Wahrnehmens, Denkens und Fühlens aufweisen (ebd.: 48). Ein solch simplifizierendes Kulturverständnis, welches Kulturen als homogene Nationalkulturen fasst, ist laut Altmayer (2006: 48) bereits seit Bestehen der Industrialisierung überholt, in Zeiten der globalen Vernetzung und des weltweiten Kulturaustausches jedoch völlig obsolet. Zwar ist im Kontext des Unterrichts positiv anzumerken, dass Lernende ihre kulturelle Prägung einbringen können, problematisch ist aber, dass dies meistens kontrastiv (die eine Kultur vs. die andere) und kulturalistische und ethnisierende Zuschreibungen reproduzierend passiert. Folglich geraten Lernende eben nicht als Individuen, sondern als Repräsentant_innen der eigenen Kultur in den Fokus interkultureller Didaktik (vgl. Altmayer 2006: 48). Das Problem einer solchen simplifizierenden Dichotomie zwischen „eigen“ und „fremd“ führt ferner dazu, dass Lernenden von außen die Zugehörigkeit zu „einer" bestimmten Kultur (fremd)zugeschrieben wird, welche darüber hinaus ihr Verhalten, Wahrnehmen und Denken prägt oder gar völlig determiniert (vgl. ebd.). Diese Sicht wird zu Recht als unwissenschaftlich und höchst problematisch beschrieben. ${ }^{5}$ In seinem bereits im Jahre 2006 erschienenen Beitrag „Kulturelle Deutungsmuster als Lerngegenstand. Zur kulturwissenschaftlichen Transformation der ,Landeskunde““, übt Claus Altmayer aber nicht nur nachvollziehbar Kritik am interkulturellen Landeskundeparadigma, sondern artikuliert gleichzeitig die Forderung nach einer Transformation der interkulturellen Landeskunde hin zu einer mit kulturwissenschaftlicher Differenzierung. Kulturwissenschaftliche Ansätze streben nach einer wissenschaftlichen Fundierung der Landeskunde und ferner danach, einen simplifizierenden Kulturbegriff, der auf die monolithische Einheit von „eine Kultur“, „eine Sprache“ und „eine Nation“ bezogen ist und Kultur demnach als homogene und abgeschlossene Größe behandelt in Frage zu stellen und durch ein differenzierteres Bild zu ersetzen. An die Stelle von kulturkontrastiven Ansätzen von „eigen“ und „fremd“ sollen bedeutungs- und diskursorientierte Konzepte treten (vgl. Altmayer: 2008). Die Auseinandersetzung mit kulturwissenschaftlichen Ansätzen soll an dieser Stelle vorerst abgebrochen und im Rahmen des Kapitels 2 Forschungsvorhaben nochmal aufgegriffen werden. Nachdem nun ein historischer Überblick über die wichtigsten landeskundlichen Entwicklungslinien gegeben wurde, wird nachfolgend das zweite zentrale Konzept Erinnerungsorte kurz illustriert.

5 Kulturalisierende Zuschreibungen schließen häufig auch an Figurationen des Kulturrassismus an (vgl. etwa Hall: 1990). 


\section{Erinnerungsorte}

Der Begriff Erinnerungsorte (lieux de mémoire) geht auf den französischen Historiker Pierre Nora zurück. Dieser wollte „Kristallisationspunkte“ des nationalen, französischen Erbes identifizieren und publik machen. Ergebnis besagter inhaltlicher Auseinandersetzung ist die siebenbändige Sammlung „Les lieux de mémoire“, (vgl. Nora, 1984-1992) die über 130 derartige Orte als Ausdruck einer „erwünschten Nationalerinnerung“ fasst und vergleichbare Projekte in vielen Ländern Europas anregte (ebd.). ${ }^{6}$

In der Sammlung „Deutsche Erinnerungsorte“ aus dem Jahre 2001 von Etienne François, und Hagen Schulze etwa wird Noras identitätsstiftende, nationale Codierung dahingehend modifiziert, dass 120 Phänomene aus verschiedenen Blickwinkeln interdisziplinär dargestellt werden, wobei versucht wird, den Begriff zu öffnen und gesamteuropäisch zu verknüpfen (vgl. Badstübner-Kizik 2011: 47). Noras Konzept wird nämlich häufig angelastet, abgegrenztes, und abgeschlossenes nationales Gedächtnis aus der begrenzten und privilegierten Autor_innen-Sicht konstruieren zu wollen (vgl. ebd.). Camilla Badstübner-Kizik verweist in ihrem Beitrag „Erinnerungsorte“ in der fremdsprachlichen Kulturdidaktik. Anmerkungen zu ihrem didaktisch-methodischen Potenzial auf die zunehmende fremdsprachendidaktische Bedeutung der Erinnerungsorte: „Auf der Suche nach authentischen, mehrdimensionalen, trag- und entwicklungsfähigen Lernanlässen und -inhalten geraten ,Erinnerungsorte“ in den letzten Jahren ins Blickfeld“ (Camilla Badstübner-Kizik 2011: 64) der didaktischen Betrachtung. Dies scheint der Autorin zufolge nur auf den ersten Blick ein Rückschritt in Richtung Renationalisierung und Kulturalisierung landeskundlichen Lernens zu sein, da EO (Erinnerungsorte) bei richtiger Auswahl gerade in homogenen Lernendengruppen Ausgangspunkt einer differenzierten Selbst- und Fremdwahrnehmung sein können (vgl. ebd.) Zwar bedeutet der Rekurs auf EO keinesfalls zwingend eine Renationalisierung, dennoch verdeutlicht das Zitat, dass im DaF-Kontext auch in Bezug auf EO noch in den 2010er Jahren - und häufig noch immer - eine (kultur)kontrastive Betrachtung vorherrscht. Gerade weil sich die Bedeutung und Wahrnehmung von Erinnerungsorten im Laufe der Zeit ändert und diese ihre gruppendistinktive Bedeutung auf ganz verschiedenen Ebenen entfalten (vgl. Badstübner-Kizik 2011: 69), will mein Dissertationsvorhaben auch das Konzept Erinnerungsorte in einer neuen, nämlich heimatkritischen, migrationssensiblen und zuschreibungsreflexiven Weise konzipieren und somit auch für migrationsgesellschaftliche Lehr- und Lernkontexte im Feld DaZ/DaF fruchtbar machen. Im anschließenden Kapitel soll nun mein Forschungsvorhaben beschrieben und in Hinblick auf seine Relevanz erklärt werden.

\section{Forschungsvorhaben}

Wenig beachtete migrationsgesellschaftliche Erinnerungsorte sollen auf eine heimatkritische Grundlage gestellt werden und damit einhergehend wird das Didaktisierungs-

6 Für den österreichischen Kontext ist hier das Werk Memoria Austriae zu nennen, das auf eine österreichweite Umfrage von 1000 Personen zurückgeht (Brix - Bruckmüller - Stekl 2004/05, Bd. 1). 
konzept einer migrationssensiblen und heimatkritischen Landeskunde für die Erwachsenenbildung DaF/DaZ generiert werden. Claus Altmayers Artikel aus dem Jahre 2017 „Landeskunde im Globalisierungskontext: Wozu noch Kultur im DaF-Unterricht?“ wirft aktuell die Frage auf, ob gängige landeskundliche Konzepte im Kontext von Deutsch als Fremdsprache in einer globalen Welt, in der Begriffe wie „Land“, „Nation“, oder „kulturelle Identität“ obsolet geworden sind, noch brauchbar und aktuell sein können (Altmayer 2017: 3). Abermals, wird hier der notwendige und sich schon längere Zeit abzeichnende Paradigmenwechsel hin zu einer kulturwissenschaftlichen Ausdifferenzierung gefordert. Bemerkenswert an dieser Momentaufnahme ist ferner, dass mit den aktuellen Tendenzen zur Standardisierung und Kompetenzorientierung eine gegenläufige Tendenz in der Praxis vorliegt, die differenzierte und divergente kulturelle Zugänge gar aus dem DaF-Unterricht zu verdrängen droht. Claus Altmayer (ebd.) formuliert die eindringliche Forderung an das Fach Deutsch als Fremdsprache, „den Zumutungen und Herausforderungen der kulturellen Globalisierung nicht auszuweichen, sondern sich offensiv mit ihnen auseinanderzusetzen“. Er skizziert ein Modell von kulturbezogenem Lernen, das den Anspruch erhebt, den Erfordernissen und Gegebenheiten einer globalisierten Welt weitaus besser gerecht zu werden als herkömmliche landeskundliche Konzepte, die noch immer einem methodologischen Nationalismus aufsitzen. Die Diagnose Methodologischer Nationalismus nach Beck - Beck-Gernsheim (2007: 236) kann auf die Landeskunde deshalb angewendet werden, weil diese in ihren interkulturellen Ausprägungen sämtliche Gegenstände von vornherein auf nationalstaatliche und territorial gegliederte Ordnungskategorien bezieht (vgl. Altmayer 2017: 9). An dieser Problematik ändert auch eine plurizentrische Perspektive nichts, weil sie zwar den germanozentrischen Fokus aufhebt und um eine österreichische bzw. schweizerische Perspektive erweitert, aber dennoch innerhalb nationalstaatlicher Ordnungskategorien operiert (vgl. ebd.). Das Modell des kulturbezogenen Lernens hat nicht ein Land oder mehrere Länder als zentralen Gegenstand, sondern vielmehr diskursive Prozesse von Bedeutungszuschreibung und Aushandlung. Ein weiteres Ziel von fremdsprachlichem, kulturwissenschaftlichem Lernen ist die Abkehr von faktischem, kulturellem Lernen. Weg vom Wissen über das „fremde Land“ oder Strategien im Umgang mit der „fremden Kultur“ und hin zur Ermächtigung und Teilhabe an bedeutungsstiftenden Diskursen (vgl. Altmayer 2017: 9). Und nicht zuletzt will sich kulturbezogenes Lernen verstanden wissen als individueller, an deutungsmusteranknüpfender Prozess (ebd.). Altmayers eingangs geschilderter Forderung an das Fach Deutsch als Fremdsprache, sich den „Herausforderungen und Zumutungen“ einer globalisierten Welt nicht zu versperren, sondern sie anzunehmen möchte ich mich mit dem Forschungsdesiderat dieser Dissertation anschließen und den Kontext Deutsch als Zweitsprache in meine Betrachtungen ebenfalls berücksichtigen. Dass eine offensive und kulturwissenschaftliche Auseinandersetzung notwendig ist, zeigen auch andere Veröffentlichungen (etwa Krumm et. al. 2010; Rösler 2012) in denen in Bezug auf Landeskunde didaktisch-methodische Aspekte im Vordergrund stehen, wohingegen kulturwissenschaftliche Fragen nur ergänzend Erwähnung finden und migrationswissenschaftliche Zugänge gänzlich unberücksichtigt bleiben. Das Dissertationsprojekt Migrationsgesellschaftliche Erinnerungsorte auf heimatkritischer Grundlage. Konturen eines 
Didaktisierungskonzeptes für die Erwachsenenbildung $D a F / D a Z$ möchte didaktisch bislang wenig beachtete und problematisierte Erinnerungsorte, wie etwa die „Ausländerbehörde“ im Rahmen der (Arbeits-)Migration, für Deutschlernkontexte fruchtbar machen und gleichzeitig Konturen einer heimatkritischen- und migrationssensiblen Landeskunde entwerfen. Auch Migrationsorte müssen als konstitutiver Bestandteil amtlich deutschsprachiger Regionen $^{7}$ sichtbar gemacht, und diese nicht mehr als monokulturell „deutschsprachige Länder“, sondern als zwar deutschsprachig dominante, aber multilinguale Migrationsgesellschaften mit kulturellen Veränderungen, die jenseits von globalen Medien gerade auch durch Migrationsprozesse angestoßen werden, gefasst werden. Erinnerungsorte sollen auch als geographische und zur Teilhabe ermächtigende Orte verstanden werden. In etwa 15 Stadtspaziergängen ${ }^{8}$ in Leipzig und Wien mit Migrant_innen und „Geflüchteten" sollen bedeutungsträchtige, migrationsgesellschaftliche-faktisch-symbolische Orte ermittelt und beschrieben werden. Methodologisch ist die Frage relevant, ob im Sinne einer migrationsgesellschaftlichen, heimatkritischen, reflexiven Landeskunde, hegemonial kulturelle Erinnerungsorte um Erinnerungsorte von Migrant_innen und Geflüchteten erweitert werden können, ohne diese als Opfer zu stigmatisieren und als homogene Gruppe zu konstituieren. Zudem muss die Frage geklärt werden, inwieweit die in den Stadtspaziergängen ermittelten Orte Erinnerungsorte im „klassischen Sinne“ sind und wie sie ihre gruppendistinktive Bedeutung entfalten. Diese Fragen werden ebenso, wie der erwartete Aushandlungsprozess um die Begriffe heimatkritisch und migrationssensibel in Kapitel 4 Erwarteter Ertrag thematisiert. Zunächst soll kurz das für mein Dissertationsvorhaben gewählte Methodendesign erklärt werden.

\section{Methodologische Überlegungen und Forschungsdesign}

Einerseits sollen in einem an migrationspädagogische Konzepte anschließenden (vgl. Mecheril et. al. 2010) Rekurs die Termini migrationssensibel und heimatkritisch definiert und als landeskundlicher Zugang fruchtbar gemacht werden und andererseits will die geplante Dissertation in ca. 15 Stadtspaziergängen in Leipzig und Wien migrationsgesellschaftliche-faktisch-symbolische Orte ermitteln und in ihrer Bedeutung für Migrant_innen und Flüchtende ${ }^{9}$ beschreiben. Warum (gerade) die Methode (Stadt-)Spaziergang als für das Forschungsdesiderat prädestiniert betrachtet wird, soll nachfolgend kurz erläutert werden. In seinem 2017 publizierten Beitrag „Postmigrantische Perspektive auf Migration, Stadt und Urbanität“ skizziert Erol Yildiz die Notwendigkeit einer postmigrantischen Perspektive, welche sich vor allem durch die drei folgenden zentralen Begriffe konstituiert:

7 Zu dieser Begrifflichkeit mehr im Kapitel 4, Erwarteter Ertrag.

8 Etwa 15, weil die genau Zahl nicht absehbar ist. Im Sinne der Grounded Theory Method werden so viele Stadtspaziergänge gemacht, bis das Material gesättigt ist.

9 Die begriffliche Aushandlung bzgl. der Frage, welcher Terminus hier angemessener erscheint, wird im Rahmen der Dissertation behandelt werden. Für den Begriff Flüchtende spricht, dass dieser kein Ankommen impliziert und markiert und dass der Zustand des Ankommens nicht als (automatisch) mit der (auch sozialen) Ankunft an einen Ort verbunden dargestellt wird. 
„Erstens geht es um die Relevanz einer Neuerzählung der ,Gastarbeitergeschichte‘. Zweitens wird der Versuch unternommen, die Migrationsforschung aus ihrer Sonderrolle zu befreien und sie als Gesellschaftsanalyse zu etablieren und drittens richtet sich die Aufmerksamkeit auf urbane Verortungspraxen von Angehörigen der zweiten und dritten Migrationsgeneration, die nicht über die gleichen Migrationserfahrungen verfügen wie ihre Eltern oder Großeltern, die aber begonnen haben, ihre eigenen Geschichten zu erzählen.“ (Yildiz 2017: 20)

Diese „urbanen Verortungspraxen“ sollen auch innerhalb der angedachten Stadtspaziergänge meines Forschungsvorhabens fruchtbar gemacht werden und bezüglich ihrer ermächtigenden Potentiale für Geflüchtete hinterfragt werden, ohne dass diese als homogene Gruppe konstruiert werden. Erol Yildiz (vgl. ebd.) plädiert dafür, das Verhältnis von Migration, Stadt und Urbanität neu zu denken, indem der Fokus auf die Betrachtung der urbanen Lebenswirklichkeit gerichtet wird - ein Zugang, dem sich auch diese Arbeit verpflichtet, wobei die Arbeitstradition des Stadtsoziologen Georg Simmel (vgl. 1995) den methodologischen Ausgangspunkt darstellt. Der Begriff Spaziergang hat seinen etymologischen Ursprung im lateinischen Wort spatiari das vom Nomen spatium, Zwischenraum, (Weg-strecke)abgeleitet ist, und so viel wie zwischen den Räumen gehen, den Raum durch das Gehen ermessen bedeutet und somit auch auf die mit dem Spazierengehen einhergehende Auseinandersetzung mit dem durchschrittenen Raum verweist (vgl. Kirchberger 2017: 19). Spazieren als eine bewusste, auf die Wegstrecke und nicht auf die Fortbewegung gerichtete Tätigkeit, hat seinen Ursprung Ende des 18. Jahrhunderts. In Abgrenzung zum repräsentativen Promenieren des Adels nach Hofzeremoniell verlieBen finanziell bessergestellte Bürger_innen die Städte, um im Spannungsfeld von Stadt und Umland ihre Natursehnsucht zu erfüllen (vgl. Legnaro 2010: 276). Der Flaneur des 19. Jahrhunderts hingegen erfreut sich nicht an der Natur, sondern vielmehr an der Stadt selbst, die er zum „Soziotop des eigenen Erlebens“ macht (ebd.: 277). Legarno beschreibt das Gehen durch die Stadt als altetablierte Methode stadtsoziologischer Erkenntnis und verweist etwa auf Louis Sébastien Mercier, den er als „Stadtsoziologe avant la lettre" bezeichnet. Mercier schafft demnach mit seinem Tableau de Paris bereits 1788 ein differenziertes Bild der französischen Metropole (vgl. Legnaro 2010: 278). Die Chicagoer Schule der Soziologie kann als Schule des Gehens bezeichnet werden, die das Gehen als soziale Tätigkeit auffasst, welche Raumerfahrung und Körperlichkeit miteinander in Bezug setzt. Ohne an dieser Stelle näher auf die inhaltlichen Differenzen verschiedener Formen stadtsoziologischen Flanierens eingehen zu können, sei dennoch kurz auf ihre Gemeinsamkeiten verwiesen. Diese sind einerseits die Veränderung des Sehens und Wahrnehmens, die durch die Bewegung selbst erzielt werden und andererseits die Annahme, dass Sehen und Bewegung einen engen Zusammenhang mit Versprachlichung aufweisen (vgl. Legnaro 2010: 278). Gehen wird als eine „Urform der Narration“ betrachtet; im Gehen erzählen Menschen gewissermaßen den durchschrittenen Raum. Lucius Burckhardt, der als Begründer der Spaziergangswissenschaft oder Promenadologie gilt, wollte eine „neue Wissenschaft“ begründen, welche einerseits die Bedingungen der Umwelt-Wahrnehmung bewusst macht und diese gleichzeitig auch erweitert. Zeichnungen, Karikaturen, oder landschaftstheoretische Aquarelle sollten ebenso wie ästhetische 
Interventionen zu dieser veränderten Umweltwahrnehmung beitragen und neue (auch inhaltliche) Freiräume schaffen (vgl. Kirchberger 2017: 22). Bei Burkhard selbst klingt diese Forderung wie folgt:

„Wir führen eine neue Wissenschaft ein: Promenadologie oder Spaziergangswissenschaft. Sie gründet sich auf die These, dass die Umwelt nicht wahrnehmbar sei, und wenn doch, dann aufgrund von Bildvorstellungen, die sich im Kopf des Betrachters bilden und schon gebildet haben." (Burkhardt 1994)

Zwar ist unstrittig, dass die Wirkung eines Ortes auf den Menschen auch wesentlich durch die bereits bestehenden Bilder im Kopf beeinflusst ist, dennoch möchte mein Dissertationsvorhaben migrationsgesellschaftliche Erinnerungsorte aufspüren und didaktisch fruchtbar machen. Somit sollen Stadtspaziergänge hier anders als von Burkhardt ursprünglich vorgeschlagen gefasst werden.

Gehen ist, wenn es als Form der empirischen Sozialforschung fungieren will, eine Methode, welche „die Erzählung des Gehens“ hörbar machen will (vgl. Legnaro 2010: 280). Realisiert soll dies im Rahmen des vorliegenden Dissertationsprojektes in ca. 15-20 Stadtspaziergängen in Gruppen und/oder einzeln werden, in welchen ich Interviews führe, um über Erfahrungen und Wahrnehmungen des Raumes zu erfahren. Dieser Vorgang ist nach Aldo Legarno (vgl. ebd.) eine hybride Methode aus teilnehmender Beobachtung und Interview, die er als „peripatetisches Interview“ bezeichnet. Dieses Verfahren bietet sich demnach an, um die Ortsempfindungen, die (nach Taylor et al. 1996) „structure of feeling“ zu ermitteln (vgl. Legnaro 2010: 280) und ist demnach auch für das vorliegende Forschungsvorhaben geeignet. Die durchgeführten Spaziergänge werden in jedem Fall als Audios festgehalten und eventuell wird der Aspekt der teilnehmenden Beobachtung auch noch als Videoaufnahme festgehalten. Hier sind technische Durchführbarkeit und vor allem auch forschungsethische Fragen zu klären. Häufig widmen sich Stadtspaziergänge entweder einer konkreten Gegend, vornehmlich einem Stadtteil oder einem Thema und sie haben zudem einen vermittelnden Charakter (vgl. Open Urban Institute, 2018). Meine Arbeit will hingegen geographische, örtliche und thematische Komponenten verbinden und hat zudem keinen vermittelnden Anspruch. Angedacht ist ferner eine kurze, gedankliche Auseinandersetzung mit den (Erinnerungs-)Orten, die dem Planungsverfahren inhaltlich vorangestellt werden. Offene, schriftlich zu beantwortende Fragen sollen eine inhaltliche Fokussierung ermöglichen und helfen das Wesen des Ortes systematisch zu erfassen ${ }^{10}$. Die Route des Spaziergangs legen die Befragten selbst fest. Weil jedoch die Gefahr routinisierter Vermeidung besteht und man als Beobachtender nicht nur teilt, was die Proband_innen sehen, sondern auch was sie nicht sehen können bzw. wollen, wird überlegt, die Teilnehmenden an Orte zu führen und sie assoziieren zu lassen (vgl. Legnaro 2010: 281). Nun, nachdem meine methodologischen

10 Die Antworten können mehrsprachig erfolgen, gegebenenfalls werden Translator_innen hinzugezogen. 
Überlegungen zum Forschungsdesign erklärt wurden, soll kurz die Perspektive migrationssensibel ${ }^{11}$ skizziert und abschließend der erwartete Ertrag geschildert werden.

\section{Migrationssensibilität und Heimatkritik}

Aus Gründen des Umfangs wird die Perspektive Heimatkritik an dieser Stelle nur in ersten Zügen definiert werden. Dieses Desiderat wird in einer folgenden Publikation behandelt. Hier sei nur kurz so viel angemerkt: Es wird im Wesentlichen der Frage nachgegangen werden, wer über den Begriff Heimat wie adressiert (vgl. Reh - Ricken 2012) wird, oder einfacher gesagt: Wer hat ein Anrecht auf Heimat und die Zugehörigkeit zu diesem symbolischen Raum und wer nicht. In diesem Zusammenhang ist auch relevant, inwiefern Heimat ein inferiorisierendes Subjektpositionierungsangebot (vgl. Rose 2012) darstellt. Nun soll aber ein migrationssensibler Zugang verortet werden. Die Perspektive Migrationssensibilität wird bezogen auf den Begriff Migrationsgesellschaft (Mecheril 2004) und ferner auf die Perspektive Migrationspädagogik (Mecheril et al. 2004) und sieht sich als inhaltliche Ergänzung/Weiterentwicklung. Um diese Zugänge verständlich zu machen, bedarf es zunächst einmal einer Klärung der Frage, auf welches Verständnis von Migration im Folgenden rekurriert wird. Migration wird als „biografisch relevante Überschreitung kulturell, juristisch, lingual und (geo-)politisch bedeutsamer Grenzen" (ebd.: 35) verstanden, mit der die Veränderung und Bestätigung des Bestehenden einhergeht. Gerade der Aspekt der Bestätigung ist bedeutsam, weil Migration häufig verkürzend auf Phänomene des Wandels reduziert wird (vgl. ebd.). Unbestritten ist hingegen, dass Migration Gegenstand vielfältiger Diskurse sowie politischer und alltagsweltlicher Auseinandersetzungen ist, wobei zwangsläufig Fragen individueller, sozialer und gesellschaftlicher Zugehörigkeit und symbolischer wie realer nationalstaatlicher Grenzen und Grenzziehungen zum Thema werden, weil über Migration Zugehörigkeitsverhältnisse problematisiert werden (vgl. ebd.). Ein migrationspädagogischer Ansatz setzt diesem Umstand Rechnung tragend voraus, dass für die Migrationsgesellschaft und ihre Bildungszusammenhänge Zugehörigkeitsverhältnisse bedeutsam sind bzw. gemacht werden (vgl. Mecheril et al. 2004: 11). Der Terminus "natio-ethno-kulturelle-Zugehörigkeit“ (ebd.: 14) ist ein Fachbegriff der Migrationspädagogik nach Paul Mecheril, der verdeutlicht, wie „diffus, unscharf und überbestimmt“ Bezeichnungen wie „deutsch“, „tschechisch“, „brasilianisch“, etc. sind, obwohl über eben diese Bezeichnungen alltägliche Unterscheidungspraxen realisiert werden (ebd.).

11 Zunächst war angedacht, beide Perspektiven, heimatkritisch und migrationssensibel in diesem Beitrag zu skizzieren und durch ein kleines Aufgabenbeispiel die didaktische Relevanz der Perspektiven zu illustrieren. Aufgrund des begrenzten Umfangs kann an dieser Stelle aber nur die Perspektive Migrationssensibilität näher erläutert werden. Die Definition von Heimatkritik und konkrete didaktische Aufgabentypologien folgen in einer zukünftigen Publikation. 
„Wenn (in Deutschland) ${ }^{12}$ von »Migrant/innen«, »Ausländern«, »Polen«, von »Migrantenkindern«, von »Deutschen« oder »Brasilianer/innen« die Rede ist, dann - so die hier formulierte These - ist in der Regel nicht allein von Kultur, Nation oder Ethnizität die Rede, sondern in einer diffusen und mehrwertigen Weise von den auch begrifflich aufeinander verweisenden Ausdrücken Kultur, Nation und Ethnizität. Der Ausdruck natio-ethno-kulturell zeigt dies an." (Mecheril et al. 2004: 14)

Nachfolgend soll nun ein exemplarischer, kurzer schematischer Überblick über Phänomene, die der migrationspädagogische Blick zu fassen sucht, gegeben werden. Ein Blickwinkel, der Fragen gleichermaßen stellen und thematisieren will, „die bedeutsam sind für eine Pädagogik unter den Bedingungen einer Migrationsgesellschaft" (Mecheril et al. 2004: 11).

$\mathrm{Zu}$ nennen sind in diesem Zusammenhang etwa:

- Formen regulärer und irregulärer Migration

- Vermischung von Sprachen und kulturellen Praktiken als Folge von Wanderungen

- Entstehung von Zwischenwelten und hybriden Identitäten

- Phänomene der Zurechnung auf Fremdheit

- Strukturen und Prozesse alltäglichen Rassismus

- Konstruktionen des Eigenen und der Fremden

- Erschaffung neuer Formen von Ethnizität

- migrationsgesellschaftliche Selbstthematisierungen: Diskurse über Migration oder »die Fremden« (Mecheril et al. 2004: 11).

Ein weiterer Schlüsselbegriff, der hier zur Klärung und Generierung einer eigenen Perspektive eingeführt werden muss, ist der Terminus Migrationsandere:

„»Migrationsandere« ist eine Bezeichnung, die, wie jede andere Bezeichnung von Personengruppen auch, pauschalisierend und festschreibend wirkt. Allerdings ist das Kunstwort »Migrationsandere« eine Bezeichnung, die das Problem der Pauschalisierung und der Festschreibung anzeigt. Denn »Migrationsandere« ist ein Wort, das zum Ausdruck bringt, dass es »Migrant/innen« und »Ausländer/innen «und komplementär »Nicht-Migrant/innen« und »Nicht-Ausländer/innen« nicht an sich, sondern nur als relationale Phänomene gibt." (Mecheril et al. 2004: 17)

Das Kunstwort „Migrationsandere“ verdeutlicht und beschreibt also Zuschreibungspraxen, durch die Migrant_innen zu Anderen oder „Geanderten“ werden und verweist zudem darauf, dass „Migrant_innen“ oder „Nichtmigrant_innen“ nicht einfach existieren, sondern nur als relationale Phänomene. Diese Zuschreibungen sind zudem nicht nur terminologisch inexakt und festschreibend, ihnen wohnt häufig auch eine gewaltvolle Praxis inne, die über einen aus der postkolonialen Theorie stammenden Terminus nach

12 Dies gilt natürlich nicht nur für Deutschland, sondern für jeden nationalen (Bildungs-)Kontext. 
Said (1978) als das Konzept Othering gefasst werden können:

„Das Konzept des Othering beschreibt einen Diskurs des Fremdmachens als eine gewaltvolle hegemoniale Praxis. Mit Hilfe dieses Konzeptes kann erläutert werden, wie das Wir durch Identifikation eines Nicht-Wirs konstituiert wird. Dieses homogene Wir symbolisiert in seiner Spannungslosigkeit eine sichere Gemeinschaft, wohingegen das Nicht-Wir fremd und bedrohlich erscheint." (vgl. Castro Varela - Mecheril 2010: 43)

Nachdem nun für die Perspektive Migrationspädagogik zentrale Konzepte und Möglichkeiten, die mit diesem Ansatz verbunden sind, beschrieben wurden, ist es wichtig, die Ziele dieses Konzeptes zu illustrieren. Die Migrationspädagogik versteht sich als Offerte des Sprechens, Denkens und Handelns, die danach strebt, Handlungs- Erfahrungs- und Denkformen zu kreieren, die weniger Macht über andere ausüben (vgl. Mecheril et al. 2004: 19).

Dieser machtkritische Zugang ist auch zentral für die Perspektive Migrationssensibilität, welche ebenso wie die Migrationspädagogik „eine notwendig reflexive, beständige fortzuentwickelnde und somit unabschließbare Praxis“ bezeichnet (ebd.). Es wird hier von der Perspektive Migrationssensibilität gesprochen, weil es sich nicht um eine bloße Begrifflichkeit handelt, sondern um eine konkrete Blickrichtung, mit der Dinge betrachtet werden.

Eine migrationssensible Landeskunde versteht sich somit als didaktisches Korrektiv gegenüber einer interkulturell ausgerichteten und kulturalisierenden Landeskunde.

Sie will eine herrschaftskritische bzw. möglichst wenig machtvolle didaktische Handlungsperspektive im Kontext - vor allem der Erwachsenenbildung - DaF/DaZ sein. Die Bezeichnung migrationssensibel ist ebenfalls ein Kunstwort und will im Sinne der Verstrickungsblindheit (Dirim 2015) darauf hinweisen, dass das Eingebundensein in machtvolle und hierarchisierende Diskurse zu einer teils nicht bemerkbaren Verstrickung in eben jene Diskurse führt, weshalb deren machtvolle Reproduktionen nicht gänzlich vermieden, aber reduziert werden können. Voraussetzung dafür ist eine reflexive, kritische und eben sensible Perspektive.

Die didaktische Handlungsofferte Migrationssensibilität will die Konstruktion homogener Gruppen und deren Opferstigmatisierung vermeiden, sie will otheringreflexiv sein und an koloniale Figurationen anschließende Konstruktionen eines Mehrheits-Wir gegenüber einem migrantischen Nicht-Wir aufdecken bzw. durch Sensibilisierung zu alternativen didaktischen Konzepten und konkreten Aufgabentypologien führen. Handlungsofferte meint in diesem Kontext eine konkrete, didaktische Handreichung, die gleichzeitig als Korrektiv auf machtvolle Perspektiven zu verstehen ist. Der Stellenwert einer rassismustheoretischen und kritischen Perspektive soll im Sinne der Migrationspädagogik (vgl. ebd.) zu einer Thematisierung rassialisierender Erfahrungen und der Schwächung von Rassismen führen. Und eine migrationssensible Fokussierung will Gesellschaften multilingual, also auch mit sprachlichen Mischungsverhältnissen, etwa dem alternierenden Sprechen samt kultureller Veränderungen fassen und hegemonialkulturelle Erinnerungen oder Reproduktionen in didaktischen Kontexten um migrationsgesellschaftliche erweitern.

Abschließend soll der erwartete Ertrag meines Dissertationsvorhabens beschrieben werden. 


\section{Erwarteter Ertrag}

Das Dissertationsvorhaben Migrationsgesellschaftliche Erinnerungsorte auf heimatkritischer Grundlage. Konturen eines Didaktisierungskonzeptes für die Erwachsenenbildung DaF/DaZ beabsichtigt die Klärung und Definition der Begriffe heimatkritisch und migrationssensibel. Damit soll die migrationspädagogische Perspektive um diese wichtigen Zugänge ergänzt werden. Die Analyse von Textkorpora verdeutlicht die Relevanz des Forschungsdesiderats, denn es konnten nahezu keine relevanten Einträge zu den genannten Begriffen gefunden werden. ${ }^{13}$ Zudem sollen bislang wenig beachtete migrationsgesellschaftliche Erinnerungsorte ermittelt und bezüglich ihrer didaktischen Potentiale befragt werden. Diese sollen nicht im Sinne nationaler Identitätsstiftung verstanden werden, sondern vielmehr im Hinblick auf ihre ermächtigende Funktion für Migrant_innen und Flüchtende, ohne diese als homogene Gruppe zu konstruieren oder als Opfer zu stigmatisieren. Auch sollen die Befragten selbst relevante Orte vorschlagen und zeigen, womit die superiorisierte Autor_innen-Sicht reduziert werden soll. Das Hauptziel und der Hauptertrag meines Dissertationsvorhabens ist das Generieren einer heimatkritischen und migrationssensiblen, sowie anwendungsbezogenen Landeskunde für den Bereich Deutsch als Fremd- und Zweitsprache in der Erwachsenenbildung.

\section{Literaturverzeichnis}

ABCD-Thesen (1990): Zur Rolle der Landeskunde im Deutschunterricht . In: ÖDaF-Mitteilungen 2. Wien: Österreichischer Verband für Deutsch als Fremdsprache/Zweitsprache, S. 26-29.

Altmayer, Claus (2006): Kulturelle Deutungsmuster als Lerngegenstand. Zur kulturwissenschaftlichen Transformation der ,Landeskunde'. In: Fremdsprachen lehren und lernen 35, S. 44-59.

Altmayer, Claus (2008): Instrumente für die empirische Erforschung kultureller Lernprozesse im Kontext von Deutsch als Fremdsprache. In: Hu, Adelheid - Byram, Michael (hrsg.) Interkulturelle Kompetenz und fremdsprachliches Lernen. Modelle, Empirie, Evaluation. Tübingen: Narr, S. 123-128.

Altmayer, Claus (2017): Landeskunde im Globalisierungskontext: Wozu noch Kultur im DaF-Unterricht? In: Haase, Peter - Höller, Michaela (hrsg.): Kulturelles Lernen im DaF/DaZ-Unterricht. Paradigmenwechsel in der Landeskunde (Materialien Deutsch als Fremdsprache, Bd. 96). Göttingen: Universitätsverlag, S. 3-22.

Badstübner-Kizik, Camilla (2011) Erinnerungsorte in der fremdsprachlichen Kulturdidaktik. Anmerkungen zu ihrem didaktisch-methodischen Potenzial. In: Mackus, Nicole - Möhring, Jupp (hrsg.): Wege für Bildung, Beruf und Gesellschaft - mit Deutsch als Fremd- und Zweitsprache. 38. Jahrestagung des Fachverbandes Deutsch als Fremdsprache an der Universität Leipzig 2011. Göttingen: Universitätsverlag, S.43-64 (= Materialien Deutsch als Fremdsprache, 87).

13 Untersucht wurde das Vorkommen der beiden Begriffe heimatkritisch und migrationssensibel im Korpus DWDS (nicht enthalten) sowie im Dereko (dt. Referenzkorpus) und bei Cosmas II ohne nennenswerten Treffer. Der Begriff „Heimat“ verzeichnete hingegen viele Treffer in allen Korpora, ebenso wie die Begriffe „Migration“ und „Kritik“. 
Brix, Emil - Bruckmüller, Ernst - Stekl, Hannes (hrsg.) (2004-2005): Memoria Austriae, Bde. 1-3, Wien: Verlagfür Geschichte und Politik.

Burckhardt, Lucius (1994): Das Zebra streifen. In: Schriftenreihe des Fachbereichs Stadtplanung, Landschaftsplanung, Universität Gesamthochschule Kassel, Bd. 20.

Christ, Herbert (1979): Landeskundeunterricht im Rahmen des Fremdsprachenunterrichts. In: Winfried Kleine (hrsg.), Perspektiven des Fremdsprachenunterrichts in der Bundesrepublik Deutschland, Frankfurt a. M. etc.: Diesterweg, S. 74-83.

Etienne François, Etienne \& Schulze, Hagen (hrsg.) (2001): Deutsche Erinnerungsorte, 3 Bände, München: C. H. Beck.

Kirchberger, Christian (2017): Gemeinsam gehen. Der Spaziergang als Beteiligungsformat in Planungsprozessen (Dipl.). Wien: Universität Wien. https://www.partizipation.at/fileadmin/media_data/Downloads/Forschungsplattform/Diplomarbeit_Kirchberger_FINAL.pdf (9. 1. 2019).

Koreik Uwe, Pietzuch Jean-Paul (2010): Entwicklungslinien landeskundlicher Ansätze und Vermittlungskonzepte. In: Krumm, Hans-Jürgen - Fandrych, Christian - Hufeisen, Britta - Riemer, Cladia (hrsg.): Deutsch als Fremd- und Zweitsprache. Ein internationales Handbuch. HSK 35.2. Berlin u.a.: De Gruyter, S. 1441-1454.

Krumm, Hans-Jürgen - Fandrych, Christian - Hufeisen, Britta - Riemer, Claudia (hrsg.) (2010): Deutsch als Fremd- und Zweitsprache. Ein internationales Handbuch (HSK 35). Berlin u.a.: De Gruyter.

Legnaro, Aldo (2010): Über das Flanieren als eine Methode der empirischen Sozialforschung Gehen - Spazieren - Flanieren. In: Sozialer Sinn, Band 11, Heft 2, S. 275-288.

Mecheril, Paul (2010): Migrationspädagogik. Hinführung zu einer Perspektive. In: Mecheril, Paul et. al. (hrsg): Migrationspädagogik. Weinheim - Basel: Beltz Verlag.

Neuner, Gerhard (1994): Fremde Welt und eigene Erfahrung - zum Wandel der Konzepte von Landeskunde für den fremdsprachlichen Unterricht. In: Neuner, Gerhard - Asche, Monika (hrsg.): Fremde Welt und eigene Erfahrung - Konzepte von Landeskunde im fremdsprachlichen Deutschunterricht (Kasseler Werkstattberichte zur Didaktik Deutsch als Fremdsprache 3). Kassel: Universität Gesamthochschule Kassel, S. 14-39.

Open Urban Institute (2019). http:/ /www.openurbaninstitute.org (11. 1. 2019).

Pelanda, Hans-Simon (2001): Landeskundlicher Ansatz. In: Helbig, Gerhard - Götze, Lutz - Henrici, Gert - Krumm, Hans-Jürgen (hrsg.): Deutsch als Fremdsprache. Ein internationales Handbuch. 1. Halbband (HSK 19/1). Berlin - New York: De Gruyter, S. 41-55.

Gerhard, Lutz Götze, Gert Henrici. Walter de Gruyter, Berlin, New York.

Pierre Nora, Pierre (hrsg.) (1984-1992): Les lieux de mémoire. 7 Bände. Paris: Gallimard; deutsch (2005): Erinnerungsorte Frankreichs. München: C. H. Beck.

Reh, Sabine - Ricken, Norbert (2012): Das Konzept der Adressierung. Zur Methodologie einer qualitativ empirischen Erforschung von Subjektivation. In: Miethe, Ingrid - Müller, Hans-Rüdiger (hrsg.): Qualitative Bildungsforschung und Bildungstheorie. Opladen - Farmington Hills: Barbara Budrich, S. 35-56.

Rösler, Dietmar (2012): Deutsch als Fremdsprache. Eine Einführung. Stuttgart: Metzler.

Rose, Nadine (2012): Migration als Bildungsherausforderung: Subjektivierung und Diskriminierung im Spiegel von Migrationsbiografien. Bielefeld: transcript.

Schumann, Adelheid (2010): Landeskunde. In: Suhrkamp, Carola (hrsg.): Metzler Lexikon Fremdsprachendidaktik. München: Metzler, S. 158-160.

Simmel, Georg (1995): Die Großstädte und das Geistesleben. In: Rammstedt, Otthein (hrsg.): Aufsätze und Abhandlungen 1901-1908.(= Gesamtausgabe). Frankfurt am Main: Suhrkamp, S. 116-131. 
Taylor, Ian - Penny, Fraser - Evans, Karen (2002): A Tale Of Two Cities: Global Change, Local Feeling and Everday Life in the North of England, Taylor \& Francis.

Weimann, Gunther - Hosch, Wolfram (1991): Geschichte im landeskundlichen Deutschunterricht. In: Zielsprache Deutsch 2, S. 134-142.

Weimann, Gunther - Hosch, Wolfram (1993): Kulturverstehen im Deutschunterricht. Ein Projekt zur Lehrerfortbildung. In: DaF. 20 (5), S. 514-523.

Yildiz, Erol (2017): Postmigrantische Perspektiven auf Migration, Stadt und Urbanität In: Riegel, Christine - Geisen, Thomas - Yildiz, Erol (hrsg): Migration, Stadt und Urbanität: Perspektiven auf die Heterogenität migrantischer Lebenswelten. Wiesbaden: Springer Fachmedien, Wiesbaden: Imprint: Springer VS, S. 19-33.

Johannes Köck, MA / koeck@mail.muni.cz

Masarykova univerzita, Filozofická fakulta, Ústav germanistiky, nordistiky a nederlandistiky; DAAD-Lektor

Arna Nováka 1, 60200 Brno, CZ

This work can be used in accordance with the Creative Commons BY-SA 4.0 International license terms and conditions (https://creativecommons.org/licenses/by-sa/4.0/legalcode). This does not apply to works or elements (such as image or photographs) that are used in the work under a contractual license or exception or limitation to relevant rights 